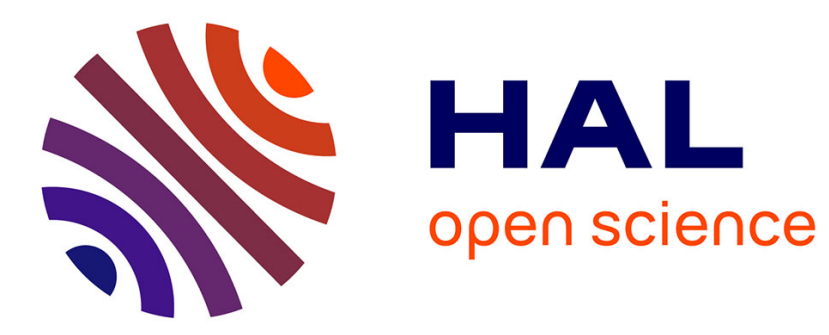

\title{
A filtered tabulated chemistry model for LES of premixed combustion
}

Benoit Fiorina, Ronan Vicquelin, Pierre Auzillon, Nasser Darabiha, Olivier Gicquel, Denis Veynante

\section{- To cite this version:}

Benoit Fiorina, Ronan Vicquelin, Pierre Auzillon, Nasser Darabiha, Olivier Gicquel, et al.. A filtered tabulated chemistry model for LES of premixed combustion. Combustion and Flame, 2010, 157 (3), pp.465-475. 10.1016/j.combustflame.2009.09.015 . hal-00472611

\section{HAL Id: hal-00472611 \\ https://hal.science/hal-00472611}

Submitted on 12 Apr 2010

HAL is a multi-disciplinary open access archive for the deposit and dissemination of scientific research documents, whether they are published or not. The documents may come from teaching and research institutions in France or abroad, or from public or private research centers.
L'archive ouverte pluridisciplinaire HAL, est destinée au dépôt et à la diffusion de documents scientifiques de niveau recherche, publiés ou non, émanant des établissements d'enseignement et de recherche français ou étrangers, des laboratoires publics ou privés. 


\title{
A filtered tabulated chemistry model for LES of premixed combustion
}

\author{
B. Fiorina ${ }^{\mathrm{a}}$, R. Vicquelin ${ }^{\mathrm{a}, \mathrm{b}}$, P. Auzillon ${ }^{\mathrm{a}}$, N. Darabiha ${ }^{\mathrm{a}}$, O. Gicquel $^{\mathrm{a}}$, D. \\ Veynante $^{\mathrm{a}}$ \\ ${ }^{a}$ EM2C - CNRS, Ecole Centrale Paris, Châtenay Malabry, France \\ ${ }^{b}$ GDF SUEZ, Pôle CHENE, Centre de Recherche et d'Innovation Gaz et Energies \\ Nouvelles, 93211 Saint-Denis la Plaine, France
}

\begin{abstract}
A new modeling strategy called F-TACLES (Filtered Tabulated Chemistry for Large Eddy Simulation) is developed to introduce tabulated chemistry methods in Large Eddy Simulation (LES) of turbulent premixed combustion. The objective is to recover the correct laminar flame propagation speed of the filtered flame front when subgrid scale turbulence vanishes as LES should tend toward Direct Numerical Simulation (DNS). The filtered flame structure is mapped using 1-D filtered laminar premixed flames. Closure of the filtered progress variable and the energy balance equations are carefully addressed in a fully compressible formulation. The methodology is first applied to 1-D filtered laminar flames, showing the ability of the model to recover the laminar flame speed and the correct chemical structure when the flame wrinkling is completely resolved. The model is then extended to turbulent combustion regimes by including subgrid scale wrinkling effects in the flame front propagation. Finally, preliminary tests of LES in a 3-D turbulent premixed flame are performed.
\end{abstract}

Key words:

Large Eddy Simulation, Turbulent premixed combustion, Tabulated chemistry

\section{Introduction}

Flame ignition and extinction or pollutant predictions are crucial issues in LES of premixed combustion and are strongly influenced by chemical effects. Unfortunately, despite the rapid increase in computational power, performing 
turbulent simulations of industrial configurations including detailed chemical mechanisms will still remain out of reach for a long time. A commonly-used approach to address fluid/chemistry interactions at a reduced computational cost consists in tabulating the chemistry as a function of a reduced set of variables. Some techniques, such as Intrinsic Low Dimensional Manifold (ILDM) developed by Mass \& Pope [1], are based on a direct mathematical analysis of the dynamic behavior of the chemical system response. Alternative approaches are Flame Prolongation of ILDM (FPI) [2, 3] or Flamelet Generated Manifold (FGM) [4]. Both techniques assume that the chemical flame structure can be described in a reduced phase subspace from elementary combustion configurations. For instance, the chemical subspace of a turbulent premixed flame can be approximated from a collection of 1-D laminar flames. In such simple situations, all thermo-chemical quantities are related to a single progress variable.

To couple tabulated chemistry with turbulent combustion, mean quantities can be estimated with presumed probability density functions. This approach, that does not require prohibitive resources, has been developed for Reynolds Averaged Navier-Stokes (RANS) computations in the past [5, 6]. Unfortunately, the extension of RANS turbulent combustion models to LES is not straightforward. Indeed, the primary recurrent problem is that the flame thickness is typically thinner than the LES grid size. As the progress variable source term is very stiff, the flame front cannot be directly resolved on practical LES grid meshes, leading to numerical issues. To overcome this difficulty, dedicated models have been developed under simplified chemistry assumptions. A solution to propagate a flame on a coarse grid is to artificially thicken the flame front by modifying the diffusion coefficient and pre-exponential constant [7, 8]. Following a different strategy and under simplified chemistry assumptions, Boger et al. [9] and more recently Duwig et al. [10] have introduced a filter larger than the mesh size to resolve the filtered flame structure. An opposite alternative is to solve a large scalar field where a given iso-surface is identified to the instantaneous flame front position. In such technique, called G-equation model, the inner layer is tracked using a level-set technique. Initially developed in a RANS context [11], the G-equation has been reformulated for LES [12, 13, 14]. However as level-set techniques provide information only on the thin reaction zone position and not on the filtered flame structure, the coupling with the flow equations is challenging. In particular the knowledge of the temperature field is required for taking into account heat expansion. As recently proposed by Moureau 
et al. under simplified chemistry assumption [15], a solution is to solve an additional progress variable equation to ensure a consistent coupling with a LES flow solver.

The FPI-PCM (Presumed Conditional Moment) model [16], developed to introduce tabulated chemistry effects in LES, combines presumed Probability Density Functions (PDF) and FPI tables to describe the chemical reaction rate of the filtered progress variable accounting for interactions between turbulence and chemistry at the subgrid scale level. However, as will be shown further, this formulation does not guarantee a proper prediction of regimes where the subgrid scale flame wrinkling vanishes. This regime, observed when the subgrid fluctuations are lower than the laminar flame speed, is encountered in practical LES of premixed combustion [17, 15]. Additionally LES should tend toward DNS when the filter size becomes lower than the Kolmogorov scale. Hawkes \& Cant [18] extensively discussed realizability in premixed combustion LES.

In the present work, it is first demonstrated that the $\beta$-PDF formalism applied in the context of premixed combustion LES does not guarantee a proper description of a filtered laminar flame front. Therefore an alternative is proposed to include tabulated chemistry in LES approach ensuring the correct propagation speed of the filtered laminar flame front. The resolved flame structure is mapped from 1-D filtered laminar premixed flames. The idea of tabulating filtered quantities has already been introduced [19] but unresolved convective and diffusive terms where neglected. As it will be demonstrated further, these assumptions do not allow a proper description of the filtered flame structure and propagation. Here, closure of filtered flow and progress variable equations are first carefully addressed in regimes where the flame wrinkling is fully resolved. One-dimensional computations are performed to investigate the capability of the proposed model to reproduce the correct propagation speed and the filtered flame structure. The model is then extended to turbulent combustion regimes taking into account subgrid scale flame wrinkling. Finally, simulations of a turbulent swirled premixed flame are performed and compared to experimental data.

\section{Coupling tabulated chemistry and LES: filtered equations}

Low-dimensional trajectories in composition space are identified in FPI framework from the knowledge of the complex chemical structure of 1-D laminar flames [2]. For premixed combustion systems, a 1-D freely propagating 
flame is first computed using detailed chemical schemes. Thermodynamical and chemical quantities are then tabulated as a function of a unique monotonic progress variable $c$ related to temperature or to a combination of chemical species, where $c=0$ corresponds to fresh gases and $c=1$ to fully burnt gases. The chemical database is then coupled to the flow field by adding the progress variable balance equation to the Navier-Stokes equations. The progress variable reaction rate and heat release are extracted from the chemical database. For LES, under unity Lewis numbers assumption, these equations are filtered leading to the following system :

$$
\begin{aligned}
\frac{\partial \bar{\rho}}{\partial t}+\nabla \cdot(\bar{\rho} \widetilde{\mathbf{u}})= & 0 \\
\frac{\partial \bar{\rho} \widetilde{\mathbf{u}}}{\partial t}+\nabla \cdot(\bar{\rho} \widetilde{\mathbf{u}} \widetilde{\mathbf{u}})= & -\nabla \bar{P}+\nabla \cdot \bar{\tau}-\nabla \cdot(\bar{\rho} \widetilde{\mathbf{u} u}-\bar{\rho} \widetilde{\mathbf{u}}) \\
\frac{\partial \bar{\rho} \widetilde{c}}{\partial t}+\nabla \cdot(\bar{\rho} \widetilde{\mathbf{u}} \widetilde{c})= & \nabla \cdot(\overline{\rho D \nabla c})-\nabla \cdot(\bar{\rho} \widetilde{\mathbf{u} c}-\bar{\rho} \widetilde{\mathbf{u}} \widetilde{c})+\bar{\rho} \widetilde{\dot{\omega}}_{c} \\
\frac{\partial \bar{\rho} \widetilde{E}}{\partial t}+\nabla \cdot(\bar{\rho} \widetilde{\mathbf{u}} \widetilde{E})= & -\nabla \cdot(\overline{P \bar{u}} \delta)+\nabla \cdot(\bar{\tau} \overline{\mathbf{u}})-\nabla \cdot(\bar{\rho} \widetilde{\mathbf{u} E}-\bar{\rho} \widetilde{\mathbf{u}} \widetilde{E}) \\
& +\nabla \cdot\left(\overline{\rho D \nabla h_{s}}\right)+\bar{\rho} \widetilde{\dot{\omega}}_{E} \\
\bar{P}= & \bar{\rho} r \widetilde{T}
\end{aligned}
$$

where $\rho$ is the density, $\mathbf{u}$ the velocity vector, $P$ the pressure, $\underline{\delta}$ the unit tensor, $\tau$ the laminar viscous tensor, $E=H-P / \rho$ with $H$ the total nonchemical enthalpy, $h_{s}$ the sensible enthalpy, $D$ is the diffusivity, $\dot{\omega}_{c}$ and $\dot{\omega}_{E}$, respectively, the progress variable and energy source terms. $r=R / W$ where $R$ is the ideal gas constant and $W$ the mean molecular weight. The overbar denotes the spatial filtering operation,

$$
\bar{\phi}(\mathbf{x})=\iiint F\left(\mathbf{x}-\mathbf{x}^{\prime}\right) \phi\left(\mathbf{x}^{\prime}\right) d \mathbf{x}^{\prime}
$$

where $\phi$ represents reactive flow variables and velocity components and $F$ the filtering function. The tilde operator denotes the density-weighted filtering defined by $\bar{\rho} \widetilde{\phi}=\overline{\rho \phi}$.

The subgrid scale terms, $-\nabla \cdot(\bar{\rho} \widetilde{\mathbf{u u}}-\bar{\rho} \widetilde{\mathbf{u}})$ and $-\nabla \cdot(\bar{\rho} \widetilde{\mathbf{u} \varphi}-\bar{\rho} \widetilde{\mathbf{u}} \widetilde{\varphi})$, where $\varphi$ denotes $c$ or $E$ quantities, the pressure term $\overline{P \mathbf{u}}$, as well as the filtered laminar diffusion terms $\overline{\rho D \nabla \varphi}$ and the filtered source terms $\widetilde{\dot{\omega}}_{\varphi}$, require closure 
models. The model constraints are both to ensure a correct flame propagation and to recover the chemical structure of the filtered flame under two possible situations : (1) the flame wrinkling is fully resolved at the LES filter size, and (2) wrinkling occurs at the subgrid scale and affects the filtered flame speed.

Different strategies exist to model the filtered progress variable reaction rate $\widetilde{\dot{\omega}}_{c}$. An approach that does not require extensive CPU resources is to presume the shape of progress variable PDF, generally by a $\beta$ function. This formalism has been applied to LES of turbulent premixed combustion [16] but, to our knowledge, the ability of the method to reproduce the propagation speed of filtered flame front has not yet been investigated. In the following section the influence of the PDF shape on the filtered flame properties is discussed when the flame wrinkling is resolved at the LES filter scale i.e. when the subgrid scale flame front remains laminar and planar. The use of a $\beta$ function is found to introduce errors in the filtered flame front propagation speed. A new modeling alternative based on the tabulation of filtered premixed flame elements is then proposed to correct this drawback.

\section{A priori testing of presumed $\beta$-PDF formalism in the laminar regime}

An unstretched 1-D filtered laminar premixed flame is considered in this section. If no wrinkling occurs at the subgrid scale, the propagation speed $S_{\Delta}$ of the filtered flame front is identical to the laminar flame speed $S_{l}^{0}$. The following relation then needs to be satisfied:

$$
\rho_{0} S_{\Delta}=\int_{-\infty}^{+\infty} \bar{\rho} \widetilde{\dot{\omega}}_{c}(x) d x=\int_{-\infty}^{+\infty} \rho \dot{\omega}_{c}(x) d x=\rho_{0} S_{l}^{0}
$$

where $\rho_{0}$ is the fresh gases density and $x$ is the spatial dimension.

The ability of presumed $\beta$-PDF to satisfy this property is investigated by conducting a priori tests on a 1-D stoichiometric freely propagating laminar premixed propane/air flame computed with PREMIX [20] using a modified version of the GRI 3.0 mechanism [21]. The progress variable $c$ is plotted as a function of the spatial coordinate $x$ in Fig. 1(a). The laminar flame thickness, defined by $\delta_{l}=1 / \max (|d c / d x|)$ is approximately equal to $0.4 \mathrm{~mm}$. Introducing $\widetilde{P}$, the mass weighted PDF defined by $\bar{\rho} \widetilde{P}=\rho \bar{P}$, the progress variable filtered reaction rate reads: 


$$
\widetilde{\dot{\omega}}_{c}(x)=\int_{0}^{1} \dot{\omega}_{c}(c) \widetilde{P}(x, c) d c
$$

Assuming that $c$ follows a $\beta$ distribution [22]:

$$
\widetilde{P}(x, c)=\frac{c^{a_{c}-1}(1-c)^{b_{c}-1}}{\int_{0}^{1} c^{a_{c}-1}(1-c)^{b_{c}-1} d c}
$$

where parameters $a_{c}$ and $b_{c}$ are determined from $\widetilde{c}$ and the segregation factor $S_{c}=(\widetilde{c c}-\widetilde{c c}) /(\widetilde{c}(1-\widetilde{c}))$ :

$$
a_{c}=\widetilde{c}\left(\frac{1}{S_{c}}-1\right) ; \quad b_{c}=a_{c}\left(\frac{1}{\widetilde{c}}-1\right)
$$

The knowledge of the first and second moment of the progress variable provides the filtered reaction rate $\widetilde{\dot{\omega}}_{c}=\widetilde{\dot{\omega}}_{c}\left(\widetilde{c}, S_{c}\right)$. For the configuration considered here, $\widetilde{c}$ and $S_{c}$ profiles across the filtered laminar flame front are computed by applying a 1-D Gaussian filter $F$ of size $\Delta$ defined by:

$$
F(x)=\left(\frac{6}{\pi \Delta^{2}}\right)^{1 / 2} e^{-\frac{6 x^{2}}{\Delta^{2}}}
$$

on the detailed chemistry laminar flame solution.

Favre-filtered progress variable and the segregation factor are shown in Fig. 1(a) for a filter size of $\Delta=20 \delta_{l}$. According to Eq. 9, the presumed $\beta$-PDF , $\widetilde{P}(x, c)$, is deduced from these two quantities. The reaction rate $\widetilde{\dot{\omega}}_{c}$ across the filtered flame front is then estimated from Eq. 8. Finally, the integration of the filtered reaction rate according to Eq. 7 gives an a priori estimation of the filtered flame front propagation speed $S_{\Delta}$. The ratio $S_{\Delta} / S_{l}^{0}$ (square symbols) is plotted as a function of the ratio $\Delta / \delta_{l}$ in Fig. 1(b). When $\Delta / \delta_{l}<1$ the effect of the $\beta$-PDF on the flame structure is moderate and the propagation speed is correctly reproduced. However when the filter size is larger than the flame front, as in LES practical situations, the propagation speed of the filtered progress variable is largely over-estimated by the presumed $\beta$ function up to a factor of 2.5 . In fact, the $\beta-\mathrm{PDF}$ is not relevant when subgrid scale wrinkling is resolved. 
A solution to propagate a flame front at the correct speed is to artificially thicken the reaction zone. In the Thickened Flame model for LES (TFLES) $[7,8]$, both reaction rate and diffusion fluxes are affected in order to ensure a correct propagation of the flame front. However the structure of the thickened flame front does not correspond to the filtered flame front.

An alternative to presumed PDF formalism and TFLES is to directly employ a normalized filter function $F(x)$ to estimate the filtered reaction rate. Then the filtered reaction rate reads:

$$
\widetilde{\dot{\omega}}_{c}(x)=\frac{1}{\bar{\rho}} \int_{-\infty}^{+\infty} \rho\left(x^{\prime}\right) \dot{\omega}_{c}\left(x^{\prime}\right) F\left(x-x^{\prime}\right) d x^{\prime},
$$

Since by definition, $F(x)$ satisfies $\int_{-\infty}^{+\infty} F(x) d x=1$, Eq. 7 is then always satisfied:

$$
\begin{aligned}
\rho_{0} S_{\Delta} & =\int_{-\infty}^{+\infty} \bar{\rho} \widetilde{\dot{\omega}}_{c}(x) d x \\
& =\int_{-\infty}^{+\infty} \int_{-\infty}^{+\infty} \rho\left(x^{\prime}\right) \dot{\omega}_{c}\left(x^{\prime}\right) F\left(x-x^{\prime}\right) d x^{\prime} d x \\
& =\int_{-\infty}^{+\infty} \rho\left(x^{\prime}\right) \dot{\omega}_{c}\left(x^{\prime}\right)\left[\int_{-\infty}^{+\infty} F\left(x-x^{\prime}\right) d x\right] d x^{\prime} \\
& =\int_{-\infty}^{+\infty} \rho\left(x^{\prime}\right) \dot{\omega}_{c}\left(x^{\prime}\right) d x^{\prime} \\
& =\rho_{0} S_{l}^{0}
\end{aligned}
$$

This property is verified in Fig. 1(b) where the propagation speed $S_{\Delta}$ of the filtered flame, is a priori computed using Eqs. 11 and 12.

By taking advantages of this property, a model is proposed in Section 4 to ensure the correct propagation of filtered laminar flame front. The closure of unknown terms is carefully addressed and the model is tested on 1-D filtered flame configurations. This approach is extended to turbulent regimes where subgrid flame wrinkling occurs at the subgrid scale level in Section 5 by the introduction of the subgrid flame wrinkling factor.

\section{Filtered laminar premixed flames modeling}

\subsection{Modeling}

The flame structure in the direction $\mathbf{n}$ normal to the flame front is assumed identical to the structure of a planar 1-D freely propagating premixed 


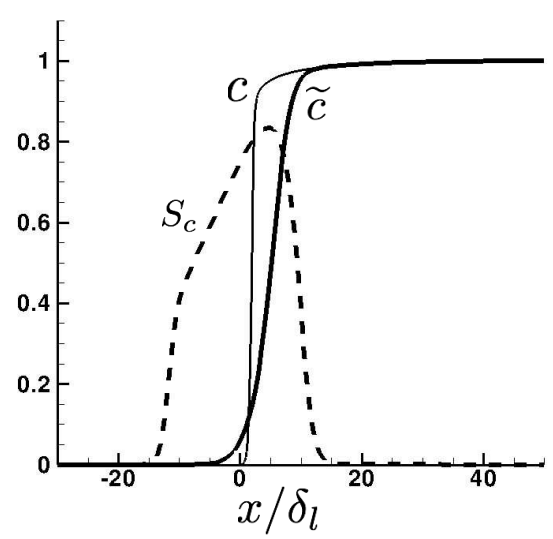

(a)

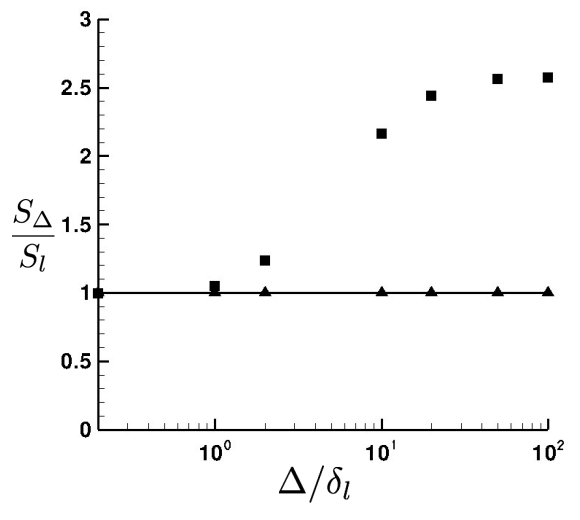

(b)

Figure 1: A priori test of the $\beta$-PDF formalism in laminar regime. Left (a): progress variable $c$ (solid line) and filtered progress variable $\widetilde{c}$ (bold line) profiles as a function of the spatial coordinate $x$. Dashed line is the subfilter progress variable segregation factor $S_{c}$. Right (b): a priori computations of the filtered progress variable propagation speed for different values of filter size. The filtered progress variable reaction rate is modeled by a $\beta$-PDF (squares) or by a Gaussian filter (triangles).

laminar flame. A detailed chemical mechanism with $N_{s}$ species is considered. From this reference flame structure and using the filter operators introduced in Section 2, the a priori filtered flame structure is determined. For instance, for a given filter size $\Delta$, any filtered fluxes or filtered thermo-chemical quantities of a planar filtered laminar flames are known.

As an example, a 1-D laminar stoichiometric premixed propane/air flame is computed taking into account detailed chemistry effects. The PREMIX [23] solver is combined with a modified version of the GRI 3.0 mechanism [21] involving $N_{s}=70$ species and 463 elementary reactions. The filtered operator given by Eq. (11) is then applied to the 1-D laminar flame solution. Figure 2 shows all budget terms of the $\widetilde{c}$ balance equation in a steady 1-D laminar premixed flame remapped in the $\widetilde{c}$ coordinate system for different values of $\Delta$. Using these results, a modeling procedure based on the tabulation of the filtered 1-D laminar flame structure is proposed in the following sections. The closure of each unclosed terms identified in Eqs. 2 to 4 is first carefully addressed in the situation where no flame wrinkling occurs at the subgrid scale level. 


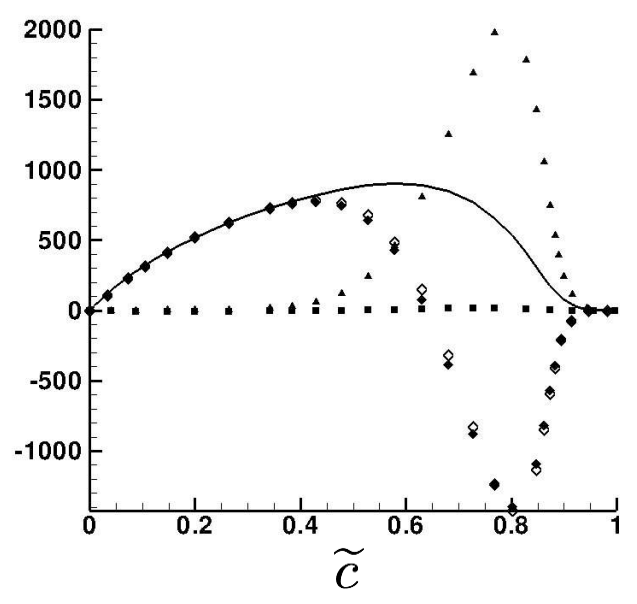

(a) $\Delta=0.2 \delta_{l}$

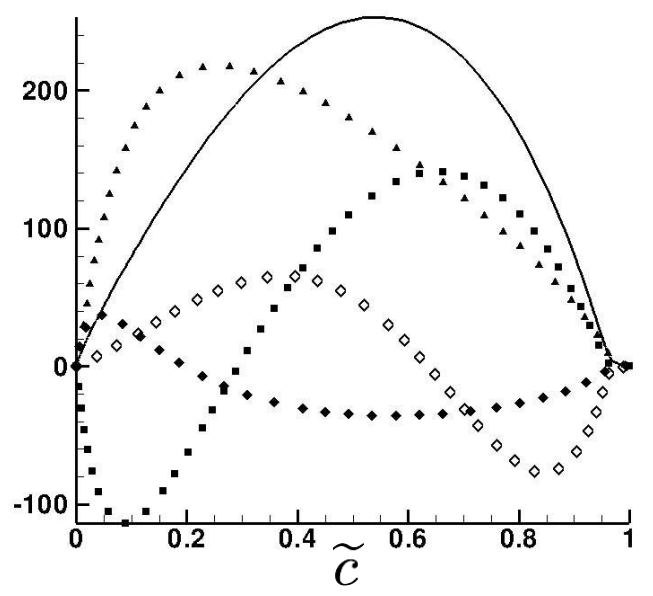

(c) $\Delta=5 \delta_{l}$

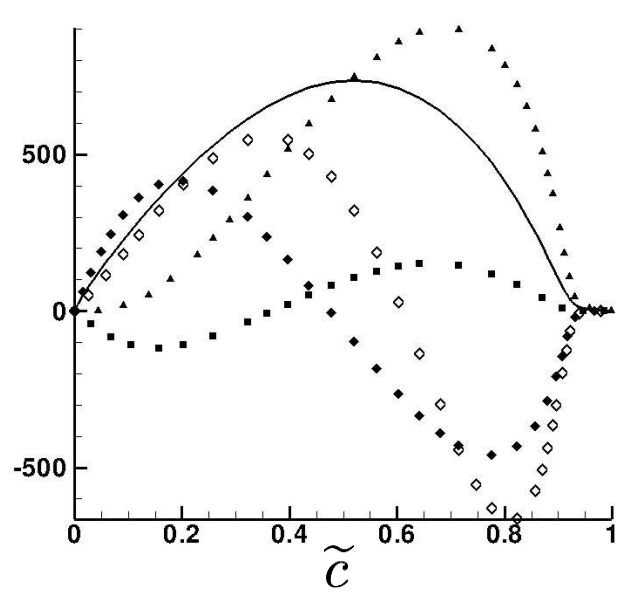

(b) $\Delta=1 \delta_{l}$

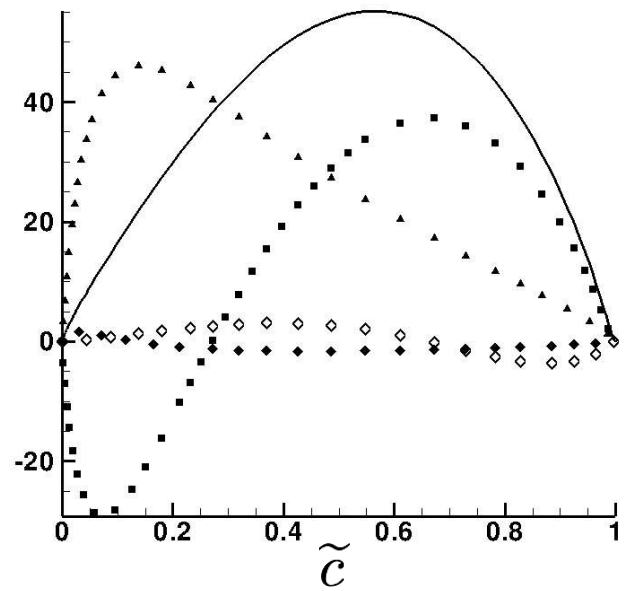

(d) $\Delta=25 \delta_{l}$

Figure 2: Budget terms (in $\mathrm{kg} \cdot \mathrm{m}^{-3} \cdot \mathrm{s}^{-1}$ ) as a function of $\widetilde{c}$ of the filtered progress variable balance equation of a steady 1-D laminar planar filtered premixed flame for different values of filter size $\Delta: \frac{\partial \bar{\rho} \widetilde{\mathbf{u}}^{*} \widetilde{c}^{*}}{\partial x^{*}}=\frac{\partial}{\partial x^{*}}\left(\overline{\rho D \frac{\partial c^{*}}{\partial x^{*}}}\right)-\frac{\partial}{\partial x^{*}}\left(\bar{\rho} \widetilde{\mathbf{u}^{*} c^{*}}-\bar{\rho} \widetilde{\mathbf{u}}^{*} \widetilde{c}^{*}\right)+\bar{\rho} \widetilde{\dot{\omega}}_{c}^{*} \cdot-\frac{\partial \bar{\rho} \widetilde{\mathbf{u}}^{*} \widetilde{c}^{*}}{\partial x^{*}}$. $\frac{\partial}{\partial x^{*}}\left(\overline{\rho D \frac{\partial c^{*}}{\partial x^{*}}}\right) \cdot \mathbf{\square}:-\frac{\partial}{\partial x^{*}}\left(\bar{\rho} \widetilde{\mathbf{u}^{*} c^{*}}-\bar{\rho} \widetilde{\mathbf{u}}^{*} \widetilde{c^{*}}\right) \cdot \mathbf{\Delta}: \bar{\rho}_{\tilde{\dot{\omega}}_{c}^{*}}^{*} \cdot \diamond: \frac{\partial}{\partial x^{*}}\left(\overline{\rho D} \frac{\partial \widetilde{c}^{*}}{\partial x^{*}}\right)$. Terms are plotted in the $\widetilde{c}$ coordinate for different values of filter size $\Delta$. 


\section{Filtered chemical reaction rates}

The filtered source terms for $c$ and the energy equations are directly given by the filtered database:

$$
\widetilde{\dot{\omega}}_{\varphi}=\widetilde{\dot{\omega}}_{\varphi}^{*}[\widetilde{c}, \Delta]
$$

where $\varphi$ denotes $c$ or $E$ quantities and the ${ }^{*}$ superscript denotes quantities issued from a 1-D unstretched laminar premixed flame. The notation $\phi[\widetilde{c}, \Delta]$ means that the variable $\phi$ is tabulated in a 2-D look-up table with coordinates $\widetilde{c}$ and $\Delta$. Figure 2 shows that the filter operator affects dramatically both the amplitude and the shape of $\widetilde{\dot{\omega}}_{c}$ (triangles symbols) profiles.

Filtered laminar diffusion terms $\nabla \cdot(\overline{\rho D \nabla c})$ and $\nabla \cdot(\overline{\rho D \nabla h})$

These terms are usually neglected or approximated as [22]:

$$
\nabla \cdot(\overline{\rho D \nabla \varphi}) \approx \nabla \cdot(\bar{\rho} D \nabla \widetilde{\varphi})
$$

This approximation is very rough and may introduce large errors. Indeed, in Fig. 2 the exact laminar diffusion fluxes $\frac{\partial}{\partial x^{*}}\left(\overline{\rho D \frac{\partial c^{*}}{\partial x^{*}}}\right)$ (filled diamonds) and the approximation by $\frac{\partial}{\partial x^{*}}\left(\bar{\rho} D \frac{\partial \widetilde{c}^{*}}{\partial x^{*}}\right)$ (empty diamonds) are shown for different values of the filter size $\Delta$. When the filter size is smaller than the laminar flame thickness $\delta_{l}$, approximation by Eq. 19 remains valid. However as soon as the filter size $\Delta$ becomes larger than $\delta_{l}$, important differences are observed between $\frac{\partial}{\partial x^{*}}\left(\overline{\rho D \frac{\partial c^{*}}{\partial x^{*}}}\right)$ and $\frac{\partial}{\partial x^{*}}\left(\bar{\rho} D \frac{\partial \widetilde{c}^{*}}{\partial x^{*}}\right)$. As shown further, these errors impact dramatically the prediction of the propagation speed. In the present work, the filtered diffusion term for the $c$ equation is modeled by:

$$
\begin{aligned}
\nabla \cdot(\overline{\rho D \nabla c}) & =-\nabla \cdot(\overline{\rho D|\nabla c|} \mathbf{n}) \\
& =-\nabla \cdot\left(\overline{\rho D\left|\frac{\partial c^{*}}{\partial x^{*}}\right|} \mathbf{n}\right)
\end{aligned}
$$

By introducing a corrective factor $\alpha_{c}(\widetilde{c})$, one can write:

$$
\nabla \cdot(\overline{\rho D \nabla c})=\nabla \cdot\left(\alpha_{c}[\widetilde{c}, \Delta] \bar{\rho} D \nabla \widetilde{c}\right) .
$$


The normal to the flame front $\mathbf{n}=-\nabla \widetilde{c} /|\nabla \widetilde{c}|$ points into the fresh reactants. The correction factor $\alpha_{c}(\widetilde{c})$ is defined as:

$$
\alpha_{c}[\widetilde{c}, \Delta]=\frac{\overline{\rho D\left|\frac{\partial c^{*}}{\partial x^{*}}\right|}}{\bar{\rho} D\left|\frac{\partial \widetilde{c}^{*}}{\partial x^{*}}\right|} .
$$

The quantity $\alpha_{c}[\widetilde{c}, \Delta]$ is estimated from the 1-D filtered flame solution and is tabulated as a function of $\widetilde{c}$ for a given value of filter size $\Delta$.

Similarly, the energy-filtered laminar diffusion term is written as:

$$
\nabla \cdot\left(\overline{\rho D \nabla h_{s}}\right)=\nabla \cdot\left(\alpha_{E}([\widetilde{c}, \Delta]) \bar{\rho} D \nabla \widetilde{h}_{s}\right),
$$

where the correction factor $\alpha_{E}[\widetilde{c}, \Delta]$ is defined as:

$$
\alpha_{E}[\widetilde{c}, \Delta]=\frac{\overline{\rho D\left|\frac{\partial h_{s}^{*}}{\partial x^{*}}\right|}}{\bar{\rho} D\left|\frac{\partial \widetilde{h}_{s}^{*}}{\partial x^{*}}\right|} .
$$

The correction factors $\alpha_{c}[\widetilde{c}, \Delta]$ and $\alpha_{E}[\widetilde{c}, \Delta]$ are plotted in Fig. 3 for different values of filter size $\Delta$. For small values of $\Delta$, as $\alpha_{c}[\widetilde{c}, \Delta]$ remains constant and close to 1 , effects on the laminar diffusion fluxes modeling will be negligible. However, the profiles present strong variations in terms of $\widetilde{c}$ when the filter size $\Delta$ is larger than $\delta_{l}$.

\section{Unresolved convection terms $-\nabla \cdot(\bar{\rho} \widetilde{\mathbf{u} \varphi}-\bar{\rho} \widetilde{\mathbf{u}} \widetilde{\varphi})$}

The displacement speed $\mathbf{s}_{\mathbf{d}}$, measuring the flame front local speed relative to the flow, i.e. the difference between the absolute flow velocity $\mathbf{u}$ and the absolute flame front speed $\mathbf{w}$, is first introduced:

$$
\mathbf{u}=\mathbf{w}+\mathbf{s}_{\mathbf{d}}
$$

The filtered flame front speed $\mathbf{w}$ remains constant across the flame brush $(\widetilde{\mathbf{w}}=\overline{\mathbf{w}}=\mathbf{w})$, therefore after replacing the flow velocity by relation 27 , the subgrid scale convection term then reads:

$$
-\nabla \cdot(\bar{\rho} \widetilde{\mathbf{u} \varphi}-\bar{\rho} \widetilde{\mathbf{u}} \widetilde{\varphi})=-\nabla \cdot\left(\bar{\rho} \widetilde{\mathbf{s}_{\mathbf{d}} \varphi}-\bar{\rho} \widetilde{\mathbf{s}_{\mathbf{d}}} \widetilde{\varphi}\right)
$$




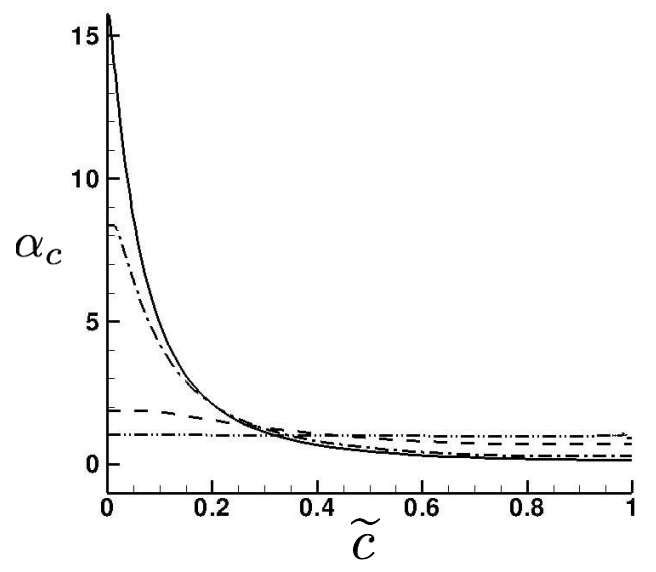

(a) $\alpha_{c}$

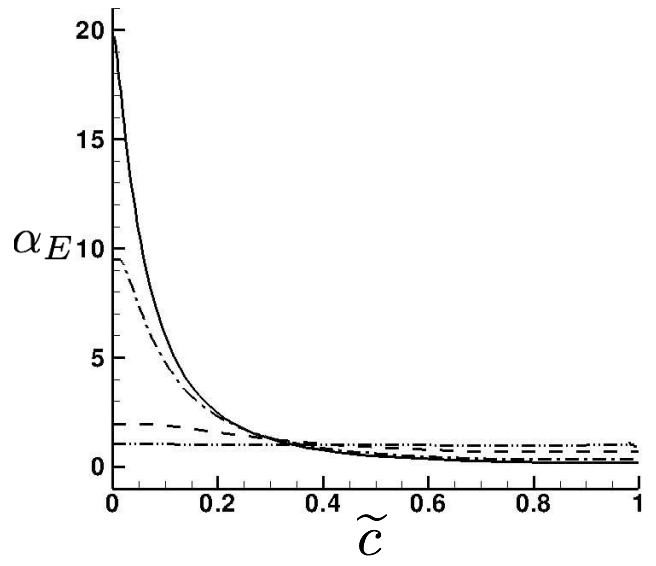

(b) $\alpha_{E}$

Figure 3: Diffusion correction factor $\alpha_{c}$ (left) and $\alpha_{E}$ (right) as a function of $\widetilde{c}$ for different values of $\Delta$. Dashed dotted dotted lines: $\Delta=0.2 \delta_{l}$. Dashed lines: $\Delta=\delta_{l}$. Dashed dotted lines: $\Delta=5 \delta_{l}$. Solid lines: $\Delta=25 \delta_{l}$.

In a 1-D laminar premixed flame the laminar flame speed $S_{l}^{0}$ and the fresh gas mixture density $\rho_{0}$ are related to the displacement speed through the following relation:

$$
\rho_{0} S_{l}^{0}=\bar{\rho} s_{d}^{*}
$$

Therefore, under the assumption that the flame remains planar at the subgrid scale level, the unresolved convection terms are directly estimated from the reference laminar 1-D detailed chemistry premixed flame:

$$
\begin{aligned}
-\nabla \cdot(\bar{\rho} \widetilde{\mathbf{u} \varphi}-\bar{\rho} \widetilde{\mathbf{u}} \widetilde{\varphi}) & =-\frac{\partial}{\partial x^{*}}\left(\bar{\rho} \widetilde{s_{d}^{*} \varphi^{*}}-\bar{\rho} \widetilde{s}_{d}^{*} \widetilde{\varphi}^{*}\right) \\
& =-\rho_{0} S_{l}^{0}\left(\frac{\partial \overline{\varphi^{*}}}{\partial x^{*}}-\frac{\partial \widetilde{\varphi^{*}}}{\partial x^{*}}\right) . \\
& =\Omega_{\varphi}[\widetilde{c}, \Delta]
\end{aligned}
$$

The term $\Omega_{c}[\widetilde{c}, \Delta]=-\rho_{0} S_{L}^{0} \frac{\partial}{\partial x^{*}}(\bar{c}-\widetilde{c})$ is plotted in Fig. 2 for different values of filter size $\Delta$ (squares). For $\Delta<\delta_{l}$, unresolved convective fluxes are very small compared to other fluxes. However, when $\Delta \geq \delta_{l}$, these fluxes 
become important and are counter-gradient type. Note that this result is in agreement with recent experiments [24]. The quantity $\Omega_{\varphi}[\widetilde{c}, \Delta]$, estimated from the 1-D filtered flame solution, is then tabulated as a function of $\widetilde{c}$ and $\Delta$. In practice, as the unresolved convective terms are modeled as a source term, only the sum $\Sigma_{\varphi}[\widetilde{c}, \Delta]=\Omega_{\varphi}[\widetilde{c}, \Delta]+\widetilde{\dot{\omega}}_{\varphi}[\widetilde{c}, \Delta]$ is stored in the filtered database where $\phi$ denotes $c$ or $E$ quantities.

\section{Pressure term}

In a similar way, the pressure term in the energy equation (Eq. 4) is written as:

$$
\begin{aligned}
-\nabla \cdot(\overline{P \underline{\mathbf{u}}} \underline{\delta}) & =-\nabla \cdot(\bar{P} \widetilde{\mathbf{u}} \underline{\delta})-(\nabla \cdot(\overline{P \underline{\mathbf{u}}} \underline{\delta})-\nabla \cdot(\bar{P} \widetilde{\mathbf{u}} \underline{\delta})) \\
& =-\nabla \cdot(\bar{P} \widetilde{\mathbf{u}} \underline{\delta})-(\nabla \cdot(\bar{\rho} \widetilde{r T} \underline{\mathbf{u}} \underline{\delta})-\nabla \cdot(\bar{\rho} \widetilde{r T} \widetilde{\mathbf{u}} \underline{\delta})) \\
& =-\nabla \cdot(\bar{P} \widetilde{\mathbf{u}} \underline{\delta})+\Omega_{p}[\widetilde{c}, \Delta]
\end{aligned}
$$

with

$$
\Omega_{p}[\widetilde{c}, \Delta]=-\rho_{0} S_{l}^{0}\left(\frac{\partial\left(\overline{r T^{*}}\right)}{\partial x^{*}}-\frac{\partial\left(\widetilde{r T^{*}}\right)}{\partial x^{*}}\right) .
$$

\section{Momentum equations}

Unclosed terms in the filtered momentum equations may be modeled following the same approach. The subgrid scale convection term is written as:

$$
\begin{aligned}
-\nabla \cdot(\bar{\rho} \widetilde{\mathbf{u}}-\bar{\rho} \widetilde{\mathbf{u}}) & =\frac{\partial}{\partial x^{*}}\left(\bar{\rho} \widetilde{s_{d}^{*} s_{d}^{*}}-\bar{\rho}{\widetilde{s_{d}}}^{*}{\widetilde{s_{d}}}^{*}\right) \mathbf{n} \\
& =\rho_{0} S_{l}^{0}\left(\frac{\partial{\widetilde{s_{d}}}^{*}}{\partial x^{*}}-\frac{\partial{\widetilde{s_{d}}}^{*}}{\partial x^{*}}\right) \mathbf{n} \\
& =\Omega_{u}[\widetilde{c}, \Delta] \mathbf{n}
\end{aligned}
$$

The strain tensor is expressed by:

$$
\nabla \cdot \bar{\tau}=\nabla \cdot\left(\alpha_{u}[\widetilde{c}, \Delta] \widetilde{\tau}\right) \quad \text { with } \quad \alpha_{u}[\widetilde{c}, \Delta]=\frac{\bar{\tau}^{*}}{\widetilde{\tau}^{*}}
$$

where $\widetilde{\tau}$ is defined as:

$$
\widetilde{\tau}=\bar{\mu}\left(\nabla \widetilde{\mathbf{u}}+(\nabla \widetilde{\mathbf{u}})^{T}-\frac{2}{3}(\nabla \cdot \widetilde{\mathbf{u}}) \underline{\delta}\right)
$$


However, as shown further, the influence of these terms is moderate and can be neglected.

\subsection{Summary of the model equations}

The momentum, the progress variable and the energy equations are modeled as:

$$
\begin{aligned}
\frac{\partial \bar{\rho} \widetilde{\mathbf{u}}}{\partial t}+\nabla \cdot(\bar{\rho} \widetilde{\mathbf{u}} \widetilde{\mathbf{u}})= & -\nabla \bar{P}+\nabla \cdot\left(\alpha_{u}[\widetilde{c}, \Delta] \widetilde{\tau}\right)+\Omega_{u}(\widetilde{c}) \mathbf{n} \\
\frac{\partial \bar{\rho} \widetilde{c}}{\partial t}+\nabla \cdot(\bar{\rho} \widetilde{\mathbf{u}} \widetilde{c})= & \nabla \cdot\left(\alpha_{c}[\widetilde{c}, \Delta] \bar{\rho} D \nabla \widetilde{c}\right)+\Sigma_{c}[\widetilde{c}, \Delta] \\
\frac{\partial \bar{\rho} \widetilde{E}}{\partial t}+\nabla \cdot(\bar{\rho} \widetilde{\mathbf{u}} \widetilde{E})= & -\nabla \cdot(\bar{P} \widetilde{u} \underline{\delta})+\Omega_{p}[\widetilde{c}, \Delta]+\nabla \cdot(\widetilde{\tau} \widetilde{\mathbf{u}}) \\
& +\nabla \cdot\left(\alpha_{E}[\widetilde{c}, \Delta] \bar{\rho} D \nabla \widetilde{h}_{s}\right)+\Sigma_{E}[\widetilde{c}, \Delta]
\end{aligned}
$$

These equations are implemented in the compressible LES code AVBP [25]. The third-order finite element scheme TTGC [26] is used. Boundary conditions are prescribed using Navier-Stokes Characteristic Boundary Conditions [27].

The sum of filtered chemical reactions rates and the subgrid scales fluxes $\Sigma_{\varphi}=\Omega_{\varphi}+\bar{\rho} \widetilde{\dot{\omega}}_{\varphi}$ and the diffusion fluxes correction factors $\alpha_{\varphi}$ are estimated after filtering a 1-D laminar stoichiometric premixed propane/air flame. These quantities are stored in a look-up table as a function of $\widetilde{c}$ and $\Delta$.

\subsection{1-D laminar premixed flame simulations}

Filtered steady 1-D laminar flames are computed to verify the ability of the present model to reproduce both the correct flame front propagation speed and the filtered flame structure. Computations are performed on uniform meshes with a grid spacing of $\Delta_{x}$. A parametric study is conducted for different filter sizes relative to the laminar flame thickness. For each case, a reference solution is obtained by filtering the 1-D laminar premixed flame detailed chemistry solution. The simulations are initialized with the reference solution and the overall physical time for each run is $t_{r u n}=50 \delta_{\widetilde{c}} / S_{l}^{0}$, where $\delta_{\widetilde{c}}=1 / \max \left(\left|\frac{\partial \widetilde{c}}{\partial x}\right|\right)$ is an estimation of the filtered flame thickness.

A comparison between the numerical solutions on uniform mesh (solid lines) and the reference solution (dashed line) with $\delta_{\tilde{c}} / \Delta_{x}=50$ and for dif-

ferent values of $\Delta / \delta_{l}$ is first shown in Fig. 4 . The predicted filtered progress 


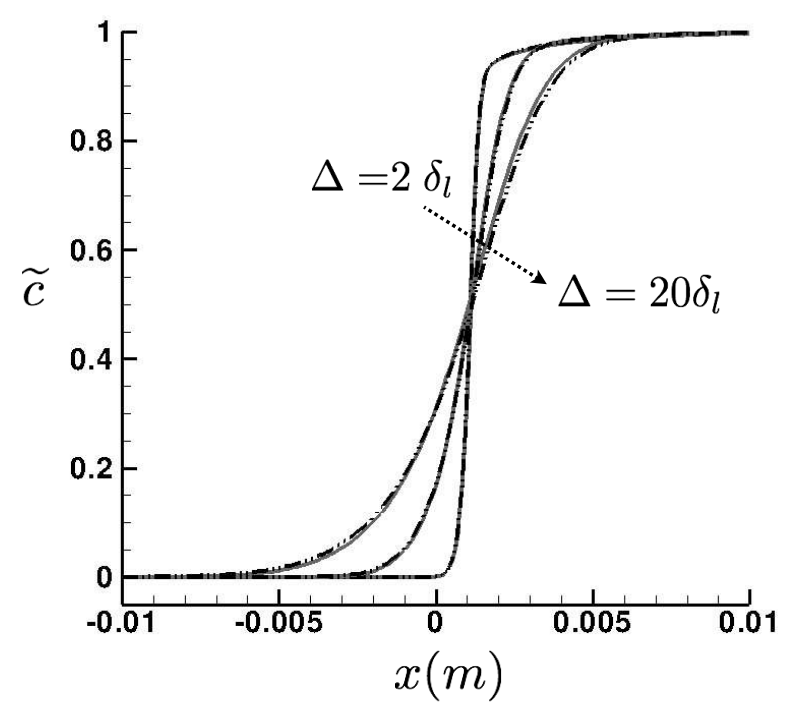

Figure 4: Filtered 1-D premixed flame solutions. Filtered progress variable (solid) compared to the reference solution (dashed) for $\Delta / \delta_{l}=2,10$ and 20.

variable profiles match the reference solution for all the filter size values. Figure 5 (a) shows that the predicted filtered front propagation speed $S_{\Delta}$ (square symbols) remains very close to the reference laminar flame speed for various values of $\Delta / \delta_{l}$. The triangular symbol in Fig. 5(a) represents simulation results with the approximation given by Eq. (19), i.e., $\alpha_{\varphi}=1$. This rough assumption leads to an under-prediction by a factor of 3 of the flame front propagation speed.

An important information for premixed combustion LES is the minimal number of grid points required to capture the filtered flame front without introducing numerical artifacts. The filtered flame front propagation speed is plotted as a function of the mesh resolution $\Delta_{x}$ in Fig.5(b). The flame speed is recovered with a good approximation for $\delta_{\tilde{c}} / \Delta_{x} \geq 5$. Below this limit, numerical errors become important and the filtered flame front does not propagate at the correct speed. Then, for numerical reasons, the filter should be at least 5 times larger than the mesh size. Note that even approaches based on level-set transport that use sophisticated numerical methods to track the flame front position also require to filter the flame front at a scale larger than the mesh size in order to resolve density gradients [15]. 


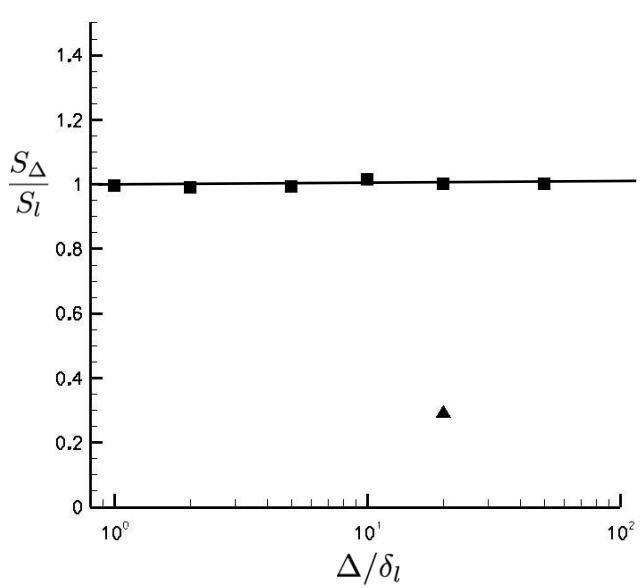

(a) $\delta_{\widetilde{c}} / \Delta_{x}=50$

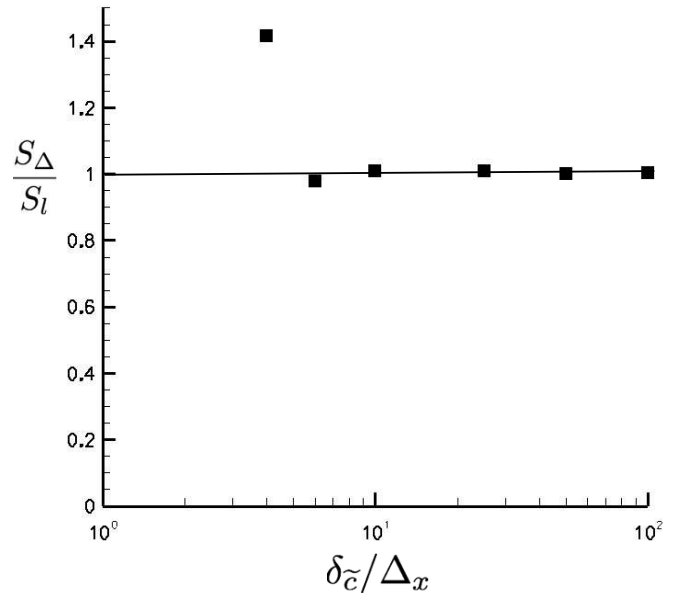

(b) $\Delta / \delta_{l}=20$

Figure 5: Predicted flame speed as a function of $\Delta / \delta_{l}$ (left) and $\delta_{\widetilde{c}} / \Delta_{x}$ (right). Square symbols are the complete model solution and the triangle symbol is the solution with $\alpha_{\varphi}=1$.

Finally, a simulation has been performed without considering the filtering effect on the momentum equations (Eq. 42), i.e., with $\alpha_{u}=1$ and $\Omega_{u}=0$ and is compared with the complete model solution in Fig. 6. For both simulations, density as well as velocity profiles match perfectly. In fact, the induced differences are transfered to the pressure that becomes a macro-pressure. As this macro-pressure remains very close to the static pressure, effects on the thermodynamic state are very limited. Then, in order to simplify the model implementation in 3-D configurations, the contribution corresponding to the filtering of a laminar flame in the momentum equation will be neglected.

\section{Filtered turbulent premixed flames modeling}

In practical LES of turbulent combustion, turbulence may cause flame front wrinkling at the subgrid scale level. Here, a strategy is proposed to extend the previously described model to such situations.

\subsection{Modeling}

Turbulent structures induce flame wrinkling that increases the flame surface area at the subgrid scale. As a consequence the filtered flame front 


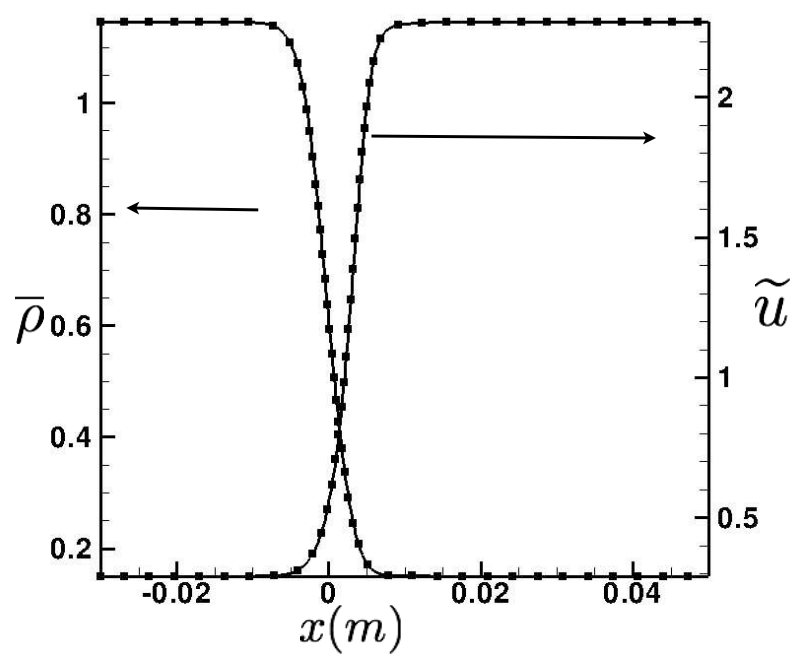

Figure 6: Filtered 1-D premixed flame solutions. Effects of the flame filter in the momentum equation. Solid: $\alpha_{u}=1$ and $\Omega_{u}=0$. Symbols: $\alpha_{u}(\widetilde{c})$ and $\Omega_{u}(\widetilde{c})$ from the filtered database.

propagates at a subgrid scale turbulent flame speed $S_{t}[22]$ related to the laminar flame speed through the flame wrinkling factor $\Xi=S_{t} / S_{l}^{0}$.

The model developed here ensures that the filtered flame front propagates at the turbulent flame speed $S_{t}$. The filtered flame thickness is assumed to be only related to the filter size $\Delta$ and is not altered by small-scale eddies.

Then, the filtered progress variable turbulent reaction rate is modeled by:

$$
\overline{\dot{\omega}}_{c_{t}}=\Xi \cdot \overline{\dot{\omega}}_{c}^{*}[\widetilde{c}, \Delta]
$$

and the turbulent diffusion term is expressed as follows:

$$
\Omega_{c_{t}}=-\left(\nabla \cdot(\bar{\rho} \widetilde{\mathbf{u}} c-\bar{\rho} \widetilde{\mathbf{u}} \widetilde{c})_{t}=\Xi \Omega_{c}[\widetilde{c}, \Delta]+(\Xi-1) \nabla \cdot\left(\alpha_{c}[\widetilde{c}, \Delta] \overline{\rho D} \nabla \widetilde{c}\right)\right.
$$

The first term on the r.h.s corresponds to the thermal expansion and the second one models the unresolved turbulent fluxes. This formulation corresponds to multiply diffusion and source terms by the flame wrinkling factor in the laminar flame balance equation and then ensures that the unstretched filtered flame front propagates at the turbulent flame speed $S_{t}=\Xi S_{l}^{0}$ in the normal direction. 


\subsection{Summary of the model equations}

To summarize, momentum, progress variable and energy equations for this new model called Filtered Tabulated Chemistry for LES (F-TACLES) can be written as follows:

$$
\begin{aligned}
\frac{\partial \bar{\rho} \widetilde{\mathbf{u}}}{\partial t}+\nabla \cdot(\bar{\rho} \widetilde{\mathbf{u}})= & -\nabla \bar{P}+\nabla \cdot \widetilde{\tau}+\nabla \cdot \bar{\tau}^{t} \\
\frac{\partial \bar{\rho} \widetilde{c}}{\partial t}+\nabla \cdot(\bar{\rho} \widetilde{\mathbf{u}} \widetilde{c})= & \Xi \nabla \cdot\left(\alpha_{c}[\widetilde{c}, \Delta] \bar{\rho} D \nabla \widetilde{c}\right)+\Xi \Sigma_{c}[\widetilde{c}, \Delta] \\
\frac{\partial \bar{\rho} \widetilde{E}}{\partial t}+\nabla \cdot(\bar{\rho} \widetilde{\mathbf{u}} \widetilde{E})= & -\nabla \cdot(\bar{P} \widetilde{\mathbf{u}} \underline{\delta})+\Xi \Omega_{p}[\widetilde{c}, \Delta]+\nabla \cdot(\widetilde{\tau} \widetilde{\mathbf{u}}) \\
& +\Xi \nabla \cdot\left(\alpha_{E}[\widetilde{c}, \Delta] \bar{\rho} D \nabla \widetilde{h}_{s}\right)+\Xi \Sigma_{E}[\widetilde{c}, \Delta] .
\end{aligned}
$$

Note that here the effect of the flame filter $\Delta$ on the momentum equations is neglected and the subgrid scale turbulent fluxes $\nabla \cdot \bar{\tau}^{t}$ are modeled using the Smagorinsky model. Different alternatives exist to estimate the subgrid flame wrinkling factor that appears in Eqs 48 and 49. It can be either estimated from analytical models $[8,14,28,29]$ or from the solution of a surface density balance equation $[30,31]$.

\subsection{Large Eddy Simulation of a swirled premixed burner}

The proposed method is applied to the simulation of the complex PRECCINSTA swirled burner experimentally investigated by Meier et al. [32]. The geometry, shown in Fig. 7, derives from an aeronautical combustion device. It features a plenum, a swirl-injector and a combustion chamber. Details of the burner geometry and of the measurement can be found in Ref. [32]. Different modeling strategies for LES have been used to numerically investigate this configuration : an LES of the combustor using the thickened flame model and a two-step mechanism has been first performed by Roux et al. [33]. Moureau et al. [34] used this configuration to validate a new level-set algorithm to track the flame front position. Recently, Galpin et al. [16] performed the LES of this lean premixed burner by using a presumed $\beta$-PDF to couple a thermo-chemical look-up table with the filtered flow equations.

The operating conditions chosen in the present study correspond to an air mass flow rate of $12.2 \mathrm{~g} / \mathrm{s}$ and to a methane mass flow rate of $0.6 \mathrm{~g} / \mathrm{s}$. In the experiment, air and methane are injected separately in the plenum inlet, however in the present simulation the mixing is assumed to be fast enough 
to burn a perfect mixing of oxidizer and fuel in the combustion chamber. Thus methane injection is not taken into account and a methane/air mixture characterized by an equivalence ratio of 0.83 is injected at the plenum inlet. These conditions correspond to a stable regime where laser Raman scattering has been performed, allowing comparison between predicted and measured thermo-chemical quantities such as temperature and species mass fractions.

The boundary conditions and the computational geometry have been already described in [33]. The mesh used to perform the computation is unstructured and made of 12.7 millions elements. The third-order finite element scheme TTGC [26] is retained. For building-up the chemical look-up table, a 1-D laminar methane/air flame is first computed for an equivalence ratio equal to 0.83 using the GRI 3.0 mechanism [21]. Then, according to the modeling procedure discussed previously, this laminar flame solution is filtered by the Gaussian function defined by Eq. 11.

Note that, as the mesh considered here is almost uniform in the filtered flame front region, an unique filter width $\Delta$ is considered. In order to ensure a sufficient meshing of the filtered flame front, the filter width has been set to $\Delta=20 \delta_{l}$. The progress variable is defined by $c=Y_{\mathrm{CO}_{2}} / Y_{\mathrm{CO}_{2}}^{e q}$, where $Y_{\mathrm{CO}_{2}}^{e q}$ is the equilibrium $\mathrm{CO}_{2}$ mass fraction in the fully burnt gases. The filtered quantities required by the model: $\Sigma_{c}[\widetilde{c}, \Delta], \alpha_{c}[\widetilde{c}, \Delta], \Omega_{p}[\widetilde{c}, \Delta], \Sigma_{E}[\widetilde{c}, \Delta]$ and $\alpha_{E}[\widetilde{c}, \Delta]$ are then tabulated as a function of $\widetilde{c}$ for $\Delta=20 \delta_{l}$. For strongly non-uniform meshes this procedure is not optimized and could lead to overrefined or under-refined flame front regions. Then, an additional coordinate, the filter width, can be easily considered when computing the look-up table.

Following the system of equations 47-49, this new model F-TACLES has been implemented into the compressible LES code AVBP [25]. The subgrid flame wrinkling factor $\Xi$ is estimated from the analytical model developed by Colin et al. [8]. Mean and resolved Root Mean Square (RMS) quantities are computed by time averaging LES solutions over a physical time that correspond to 6 flow-through times based on the fresh gas inlet velocity. Mean temperature and $\mathrm{CO}_{2}$ mass fractions are plotted on Figs. 8 (top) and 9 (top), respectively. A very good agreement is observed between experimental and numerical profiles, which demonstrated that the correct flame angle and mean flame thickness are reproduced by the model. Because heat losses have not been considered when generating the chemical database and in the numerical simulation, the LES slightly over-estimates the temperature profiles close to the combustion chamber wall, in the outer recirculation zone for $x<20 \mathrm{~mm}$ and at a distance larger than $20 \mathrm{~mm}$ from the jet axis. Note that heat losses 
effects on the flame structure can be taken into account with the addition of the enthalpy as a control parameter of the chemistry tabulation [3, 6].

Figs. 8 (bottom) and 9 (bottom) show a comparison between resolved LES RMS and measured RMS of the temperature and the $\mathrm{CO}_{2}$ mass fraction, respectively. As the plotted LES RMS does not include the subgrid scale RMS, conclusions regarding the model performance in terms of flame turbulence interactions are more difficult. However, it is observed that LES RMS remains lower than measured RMS, as expected from theory.

As all thermo-chemical variables are related to $\widetilde{c}$, the post-processing of the filtered progress variable solution with the filtered chemical database allows to access all chemical species. As an example, Fig. 10(a) shows 2-D contours of $\widetilde{c}$ used to estimate HCO mass fraction plotted in Fig. 10(b).

Finally, Fig. 11 indicates the flame position in the Pitsch LES regime diagram for turbulent premixed combustion [17], where the ratio $\Delta / \delta_{l}$ is expressed as a function of the Karlovitz number $K a$ in logarithmic scale. The Karlovitz number is related in LES to the subgrid velocity fluctuations $v_{\Delta}^{\prime}$ and laminar flame scales [17]:

$$
K a^{2}=\frac{\delta_{l}}{S_{l}^{0^{3}}} \varepsilon=\frac{v_{\Delta}^{\prime}}{S_{l}^{0}} \frac{\delta_{l}}{\Delta}
$$

where $\varepsilon$ is the kinetic energy transfer rate. The subgrid velocity fluctuations are computed as follows:

$$
v_{\Delta}^{\prime}=\frac{\mu_{t}}{\bar{\rho} C_{k} \Delta \sqrt{3 / 2}}
$$

where the turbulent viscosity $\mu_{t}$ is estimated from Smagorinsky model. For $K a<1$, combustion takes place in the corrugated flame regime while the thin reaction zone regime is observed when $K a>1$. Computational nodes located in the filtered flame front are considered, i.e. for $0.01<\widetilde{c}<0.99$, and are plotted in the LES diagram (horizontal thick solid black line in Fig. 11). As a a unique filter width $\Delta$ is considered in the present simulation, the scatter plot reduced to the line $\Delta / \delta_{l}=20$. The smallest size of the flame wrinkling is given by the Gibson length [11]:

$$
\frac{\Delta}{l_{G}}=\frac{v_{\Delta}^{\prime}}{S_{l}^{0}}
$$

The substitution of Eq. 52 into Eq. 50 shows that $\Delta=l_{G}$ condition corresponds to $\Delta / \delta_{l}=K a^{-2}$ represented by a line of slope -2 in the LES 


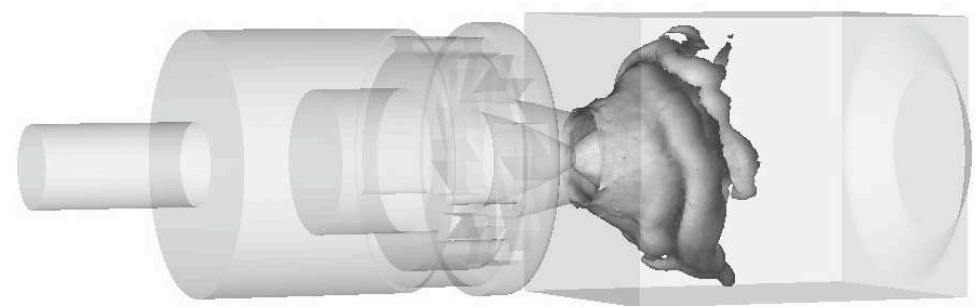

Figure 7: LES of Preccinsta with F-TACLES turbulent combustion model. The computational domain features the plenum, the swirl-injector and the combustion chamber. An instantaneous view of the filtered flame front iso-surface $(\widetilde{c}=0.8)$ is shown.

diagram (Fig. 11). In the corrugated flame regime, when the filter width becomes smaller than the Gibson length, the subgrid velocity fluctuation $v_{\Delta}^{\prime}$ is smaller than the laminar flame speed $S_{l}^{0}$. In such cases, the flame wrinkling is fully resolved at the LES filter scale. At the opposite, on the right side of the $l_{G}=\Delta$ line, subgrid scale wrinkling exists and will impact the filtered flame front propagation speed $S_{\Delta}$. The node distribution versus the Karlovitz number is plotted in Fig. 12. First, it can be observed that most of the points are located in the corrugated flame regime $(K a<1)$. The chemical flame structure remains therefore laminar as assumed in the present model. Secondly, for a substantial area of the flame surface ( about $30 \%$ ), the Gibson length $l_{G}$ is larger than the filter width and consequently the flame wrinkling is fully resolved. With future increase of computational power, as meshes will be finer, this trend should be emphasized. It demonstrates the crucial need of ensuring a proper propagation of the laminar flame front when deriving a turbulent combustion model.

\section{Conclusion}

A new modeling strategy called Filtered Tabulated Chemistry for LES (FTACLES) has been developed to introduce tabulated chemistry methods in premixed combustion LES. A filtered 1-D laminar premixed flame is used to build a filtered chemical look-up table. The model performances are demonstrated on 1-D filtered laminar flame computations. Finally the proposed strategy has been applied to perform a 3-D simulation of a swirled turbulent 

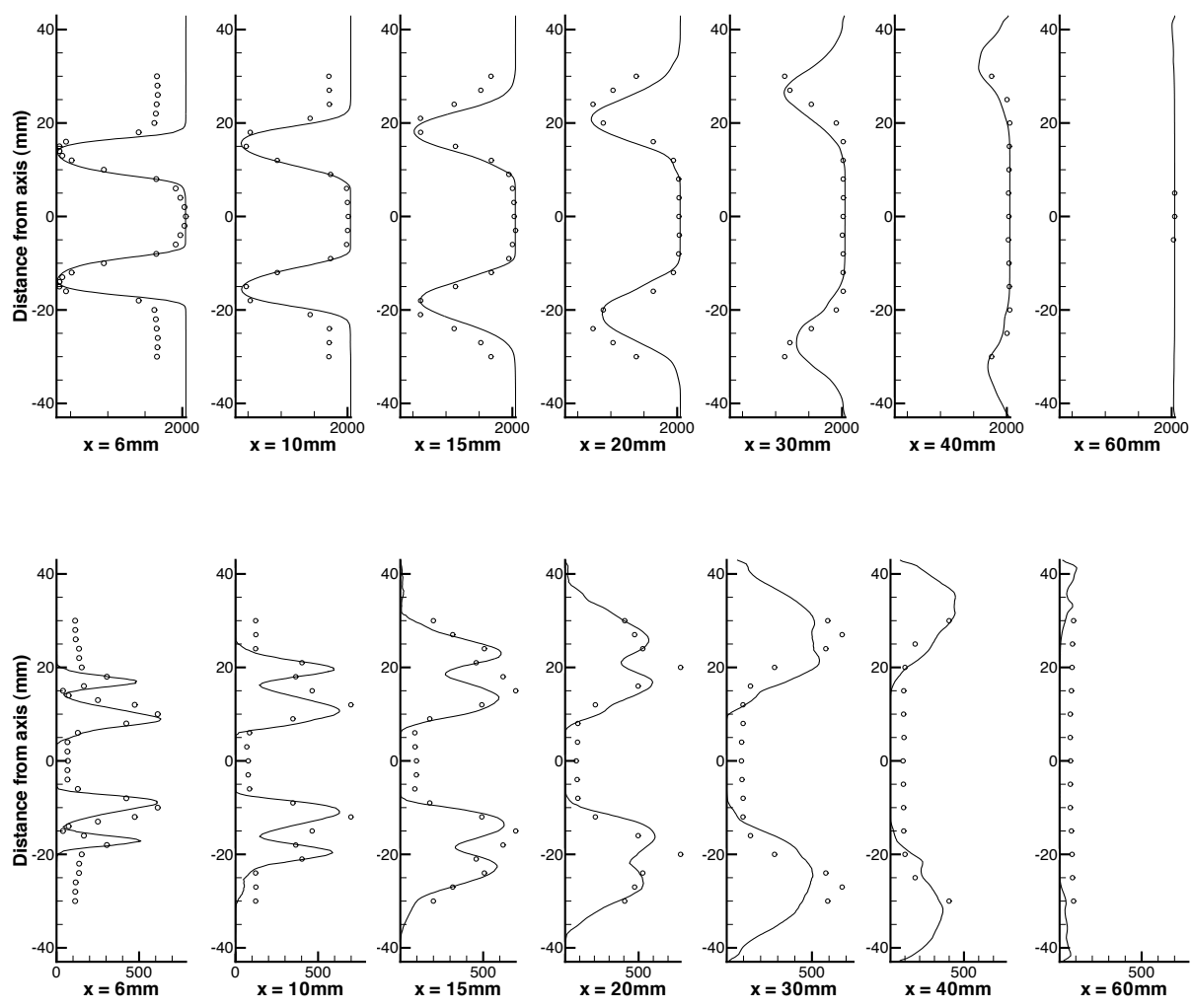

Figure 8: Mean (top) and RMS (bottom) of temperature, case $\phi=0.83$. Symbols: measurements. Lines: simulation with F-TACLES. $x=0$ matches the swirler exit. 

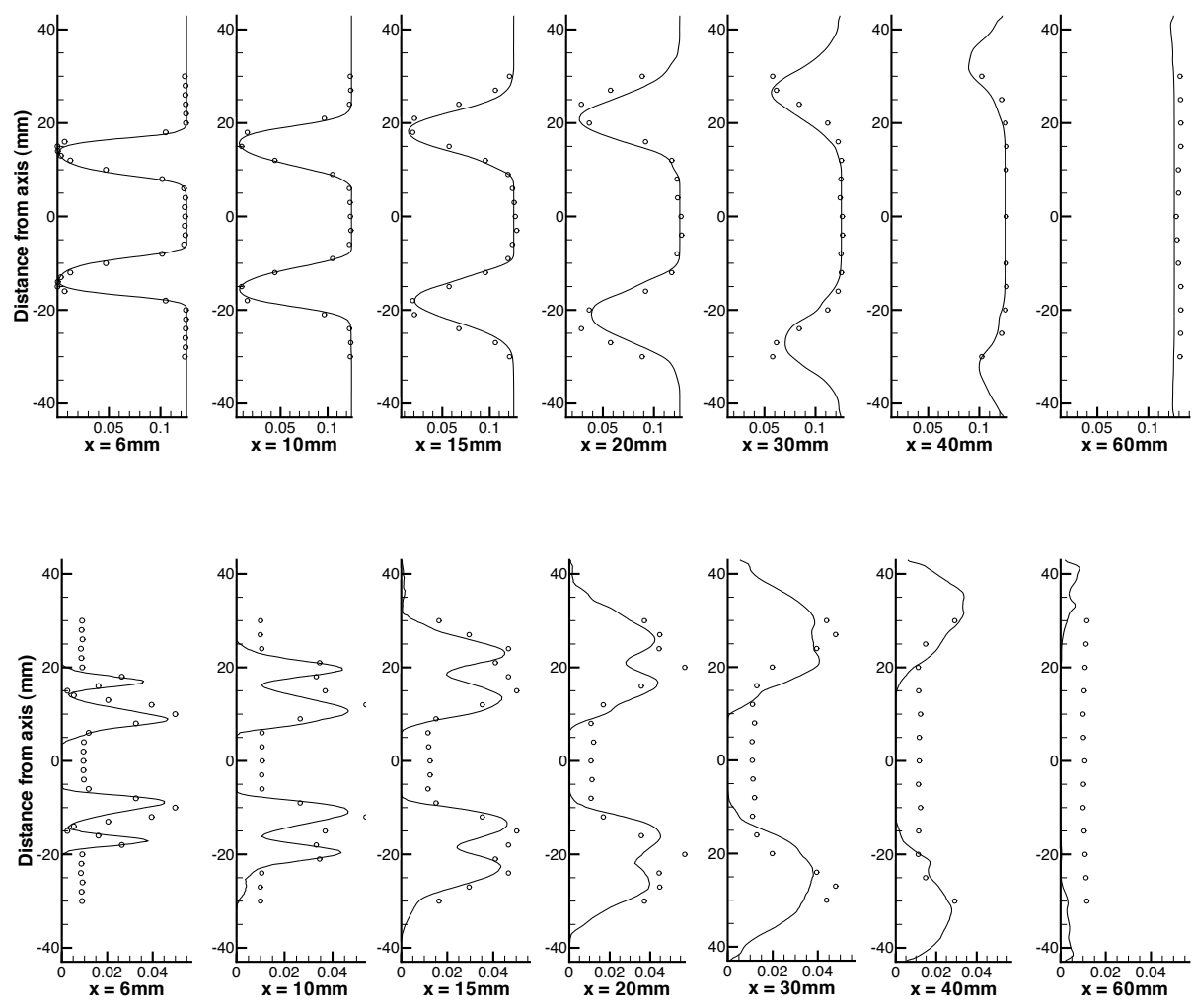

Figure 9: Mean (top) and RMS (bottom) of $\mathrm{CO}_{2}$ mass fraction, case $\phi=0.83$. Symbols: measurements. Lines: simulation with F-TACLES. $x=0$ matches the swirler exit. 

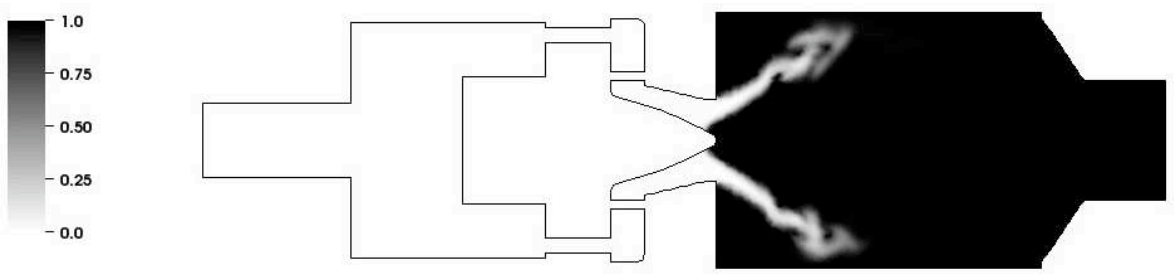

(a) $\widetilde{c}$
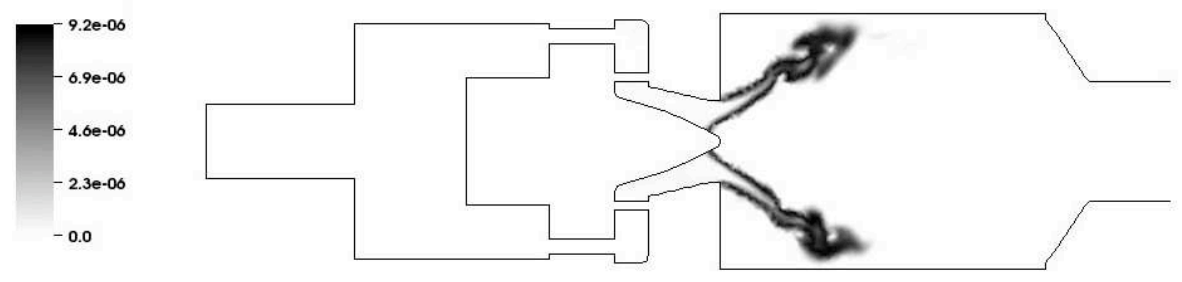

(b) $\widetilde{Y}_{H C O}$

Figure 10: 2-D instantaneous view of $\widetilde{c}$ and $\widetilde{Y}_{H C O}$.

premixed flame. Good agreement between the numerical simulation and the experiments is observed.

\section{Acknowledgments}

This work was supported by the ANR-07-CIS7-008-04 Grant of the French Ministry of Research. We are grateful to the CERFACS (Toulouse, France) combustion team for providing the PRECCINSTA burner geometry. The authors warmly acknowledge the support of the 2008 Summer Program of the Center for Turbulence Research (Stanford University - NASA Ames) during which this work was initiated. This work was granted access to the HPC resources of IDRIS under the allocation 2009-i2009020164 made by GENCI (Grand Equipement National de Calcul Intensif) 


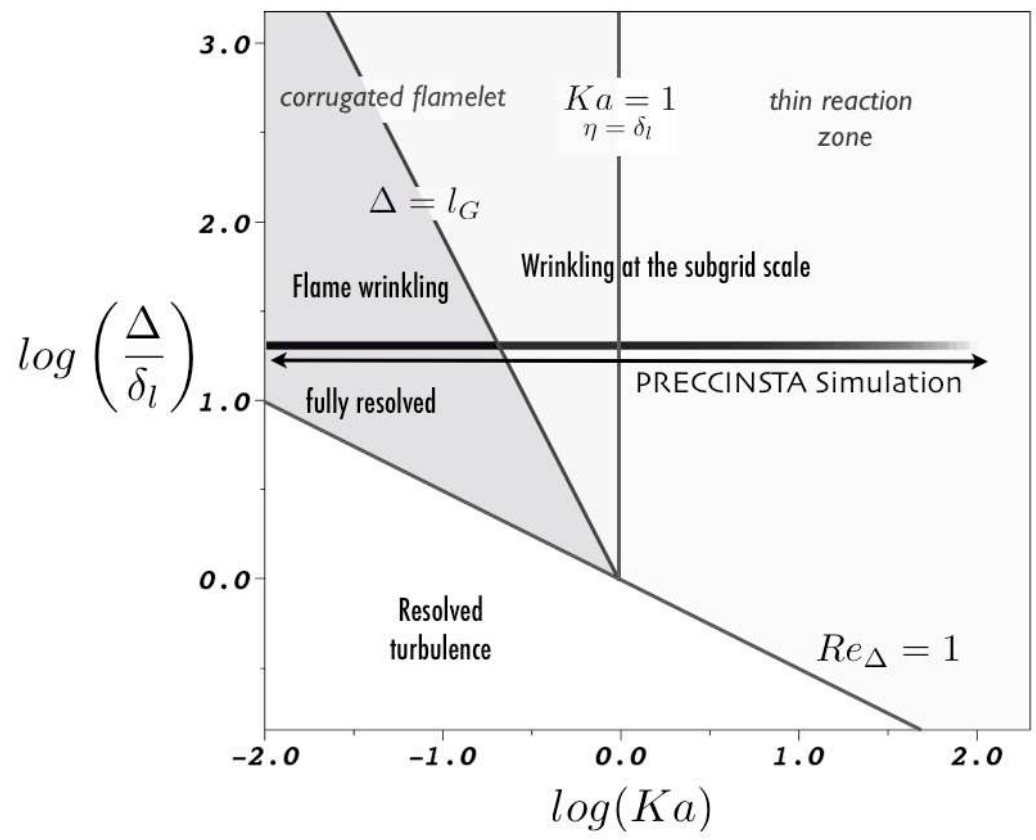

Figure 11: LES regime diagram for turbulent premixed combustion. The thick solid black line represent the range covered by the Preccinsta flame simulation. 


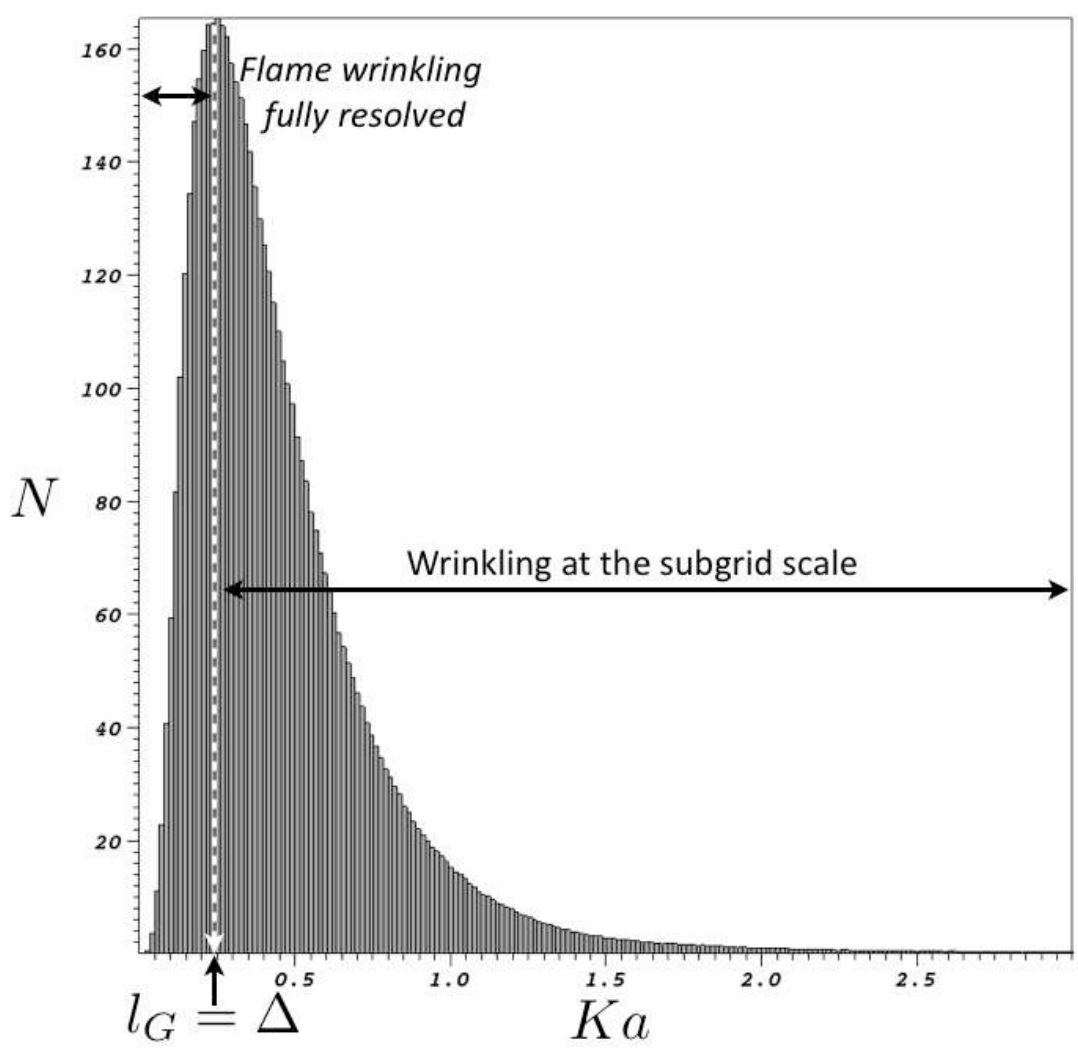

Figure 12: Node distribution versus the Karlovitz number. Only nodes located into the filtered flame front have been considered, i.e. for $0.01<\widetilde{c}<0.99$. 


\section{References}

[1] U. Maas, S. Pope, Simplifying chemical kinetics: Intrinsic lowdimensional manifolds in composition space, Combust. Flame 88 (1992) 239-264.

[2] O. Gicquel, N. Darabiha, D. Thévenin, Laminar premixed hydrogen / air counterflow flame simulations using flame prolongation of ildm with differential diffusion, in: The Proceedings of the Twenty-Eighth Symposium (Int.) on combustion. The Combustion Institute, Pittsburgh, 2000, pp. 1901-1908.

[3] B. Fiorina, R. Baron, O. Gicquel, D. Thévenin, S. Carpentier, N. Darabiha, Modelling non-adiabatic partially-premixed flames using flame prolongation of ildm, Combustion Theory and Modelling 7 (2003) 449470.

[4] J. A. van Oijen, F. A. Lammers, L. P. H. de Goey, Modelling of complex premixed burner systems by using flamelet-generated manifolds, Combust. Flame 127 (3) (2001) 2124-2134.

[5] L. Vervisch, R. Haugel, P. Domingo, M. Rullaud, Three facets of turbulent combustion modelling: Dns of premixed flame, les of lifted nonpremixed v-flame and rans of jet-flame, J. of Turbulence 5 (4) (2004) $1-36$.

[6] B. Fiorina, O. Gicquel, L. Vervisch, S. Carpentier, N. Darabiha, Premixed turbulent combustion modelling using tabulated chemistry and pdf, Proc. Combust. Inst. 30 (2005) 867-874.

[7] T. D. Butler, P. J. O'Rourke, A numerical method for two-dimensional unsteady reacting flows., Proceedings of the 16th Symp. (Int.) on Combustion. The Combustion Institute: Pittsburgh, Penn. (1977) 15031515 .

[8] O. Colin, F. Ducros, D. Veynante, T. Poinsot, A thickened flame model for large eddy simulations of turbulent premixed combustion, Physics of Fluids 12 (7) (2000) 1843-1863. 
[9] M. Boger, D. Veynante, H. Boughanem, A. Trouvé, Direct numerical simulation analysis of flame surface density concept for large eddy simulation of turbulent premixed combustion, in: Twenty-Seventh Symposium (Int.) on Combustion. The Combustion Institute: Pittsburgh, Penn., 1998, pp. 917 - 925.

[10] C. Duwig, Study of a filtered flamelet formulation for large eddy simulation of premixed turbulent flames, Flow Turbulence and Combustion 79 (4) (2007) 433-454.

[11] N. Peters, Turbulent Combustion, Cambridge University Press, 2000.

[12] S. Menon, W. Jou, Large eddy simulations of combustion instability in an axisymetric ramjet combustor, Combust. Sci. and Tech. 75 (1991) $53-72$.

[13] V.-K. Chakravarthy, S. Menon, Subgrid modeling of turbulent premixed flames in the flamelet regime, Flow, Turbulence and Combustion 65 (2000) 133-161.

[14] H. Pitsch, A consistent level set formulation for large-eddy simulation of premixed turbulent combustion, Combust. Flame 143 (4) (2005) 587598.

[15] V. Moureau, B. Fiorina, H. Pitsch, A level set formulation for premixed combustion les considering the turbulent flame structure, combust. and Flame 156 (4) (2009) 801-812.

[16] J. Galpin, A. Naudin, L. Vervisch, C. Angelberger, O. Colin, P. Domingo, Large-eddy simulation of a fuel-lean premixed turbulent swirl-burner, Combust. Flame 155 (2008) 247-266.

[17] H. Pitsch, Large-eddy simulation of turbulent combustion, Ann. Rev. Fluid Mech. 38 (2006) 453-482.

[18] E. R. Hawkes, R. S. Cant, Physical and numerical realizability requirements for flame surface denisty approaches and reynolds averaged simulation of premixed turbulent combustion, Combust. Theory and Modelling 5 (2001) 699-720. 
[19] A. W. Vreman, J. A. van Oijen, L. P. H. de Goey, R. J. M. Bastiaans, Subgrid scale modeling in large eddy simulation of turbulent combustion using premixed flamelet chemistry, Flow, Turbulence and Combustion 82 (2009) 511-535.

[20] R. J. Kee, J. F. Grcar, M. D. Smooke, J. A. Miller, A fortran program for modelling steady laminar one-dimensional premixed flames, Tech. Rep. SAND85-8240-UC-401, Sandia National Laboratories (1985).

[21] http://www.me.berkeley.edu/gri-mech/.

[22] T. Poinsot, D. Veynante, Theoretical and Numerical Combustion, R. T. Edwards, Inc., 2005.

[23] R. J. Kee, J. F. Grcar, M. D. Smooke, J. A. Miller, A fortran program for modelling steady laminar one-dimensional premixed flames, Tech. rep., Sandia National Laboratories (1992).

[24] S. Pfadler, J. Kerl, F. Beyrau, A. Leipertz, A. Sadiki, J. Scheuerlein, F. Dinkelacker, Direct evaluation of the subgrid scale scalar flux in turbulent premixed flames with conditioned dual-plane stereo piv, Proceedings of the Combustion Institute 32 (2) (2009) 1723 - 1730.

[25] Avbp code : www.cerfacs.fr/cfd/avbp code.php and www.cerfacs.fr/cfd/ cfdpublications.html.

[26] O. Colin, M. Rudgyard, Development of high-order taylor-galerkin schemes for unsteady calculations, J. Comput. Phys. 162 (2) (2000) 338371.

[27] T. Poinsot, S. K. Lele, Boundary conditions for direct simulations of compressible viscous flows, J. Comput. Phys. 1 (101) (1992) 104-129.

[28] F. Charlette, C. Meneveau, D. Veynante, A power-law flame wrinkling model for les of premixed turbulent combustion, part i: non-dynamic formulation, Combust. Flame 131 (1/2) (2002) 159-180.

[29] F. Charlette, C. Meneveau, D. Veynante, A power-law flame wrinkling model for les of premixed turbulent combustion, part ii: dynamic formulation, Combust. Flame 131 (1/2) (2002) 181-197. 
[30] E. R. Hawkes, R. S. Cant, A flame surface density approach to largeeddy simulation of premixed turbulent combustion, Proc. Combust. Inst 28 (2000) 51-58.

[31] S. Richard, O. Colin, O. Vermorel, A. Benkenida, C. Angelberger, D. Veynante, Towards large eddy simulation of combustion in spark ignition engines, Proc. Combust. Inst 31 (2007) 3059-3066.

[32] W. Meier, P. Weigand, X. Duan, R. Giezendanner-Thoben, Detailed characterization of the dynamics of thermoacoustic pulsations in a lean premixed swirl flame, Combustion and Flame 150 (1-2) (2007) 2 - 26.

[33] S. Roux, G. Lartigue, T. Poinsot, U. Meier, C. Berat, Studies of mean and unsteady flow in a swirled combustor using experiments, acoustic analysis, and large eddy simulations, Combustion and Flame 141 (1-2) (2005) $40-54$.

[34] V. Moureau, P. Minot, H. Pitsch, C. Berat, A ghost-fluid method for large-eddy simulations of premixed combustion in complex geometries, Journal of Computational Physics 221 (2) (2007) 600 - 614. 